
\title{
$\angle$ Research Square \\ Multiple Negative of RT-PCR Testing of COVID-19 Pneumonia: A Case Report
}

\section{Huili}

Xiangya Hospital Central South University

Junton Feng

Xiangya Hospital Central South University

Lamei Chen

Kaifu District Center For Discase Control and Prevention

\section{Yinhao Wu}

Xiangya Hospital Central South University

\section{Yan Su}

Xiangya Hospital Central South University

YingLi

Xiangya Hospital Central South University

Yongjun Tang ( $\nabla$ tyj7736@163.com )

Xiangya Hospital Central South University https://orcid.org/0000-0002-7526-1112

\section{Case Report}

Keywords: 2019 novel coronavirus, reverse transcriptase polymerase chain reaction test, COVID-19 pneumonia, case report

Posted Date: March 9th, 2020

DOI: https://doi.org/10.21203/rs.3.rs-16094/v1

License: (c) (i) This work is licensed under a Creative Commons Attribution 4.0 International License. Read Full License 


\section{Abstract}

Background: Since the 2019 novel coronavirus outbreak in Wuhan, Hubei Province, China in 2019, there have been a few reports of multiple negative of RT-PCR tests in patients infected by 2019 novel coronavirus.

Case presentation: The patient was a 64-year-old man with fever. His son returned from Hubei 17 days before the patient had fever. Ancillary examinations indicated a decreased lymphocyte count and groundglass opacities in lung. However, the first five reverse transcriptase polymerase chain reaction tests of 2019 novel coronavirus were negative until the sixth turned to positive.

Conclusion: when epidemiological history, clinical manifestation and imaging are highly suggestive of COVID-19 Pneumonia, we should repeat reverse transcriptase polymerase chain reaction tests and treat patients in isolation.

\section{Background}

Since December 2019, a cluster of cases of pneumonia with unexplained causes has appeared in Wuhan, Hubei Province, China. On January 2020, the pathogen was identified as a novel coronavirus, which was tentatively named by the World Health Organization(WHO) as the 2019 novel coronavirus (2019-nCoV) ${ }^{[1]}$. Subsequently, this disease was referred to as 2019 novel coronavirus disease (COVID-2019) ${ }^{[1]}$. It rapidly spread, resulting in an epidemic outbreak in China, with sporadic cases reported globally. As of February 20, 2020, 74,667 cases were confirmed, with 2,118 deaths in China. There have been a few reports about multiple negative results of reverse transcriptase polymerase chain reaction (RT-PCR) tests in patients infected by 2019- nCoV. In our report, the patient was finally found to be positive by the sixth RT-PCR 2019nCoV nucleic acid assay.

\section{Case Report}

A 64-year-old man presented with fever,dry cough, running nose and muscle aches for 8 days and was admitted to the emergency department of Xiangya Hospital. The patient was hospitalized for constrictive pericarditis and polyserosal fluid from January 14 to 22,2020 . Although the patient had not visited Wuhan recently, he had a history of close contact with an individual who had returned from Jingzhou, Hubei Province. Laboratory examination results showed a normal leukocyte count $\left(5.8 \times 10^{\wedge} 9 / \mathrm{L}\right)$ and decreased lymphocytes count $\left(0.8 \times 10^{\wedge} 12 / L\right)$. The chest CT showed ground-glass opacities in left upper lung (Fig.1). The first two oropharyngeal swab tests of 2019-nCoV were both negative after the positive CT scanning. Subsequently, 3 nasopharyngeal and oropharyngeal swab tests were all negative, but his symptoms continually aggravated. Repeated CT chest showed progression of ground-glass opacities in left upper lung and new areas of ground-glass opacities in right upper lobe and right lower lobe (Fig.2). Fifteenth days after admission, the sixth nasopharyngeal swab test was finally positive. However, oropharyngeal swab test was still negative. The patient was then transferred to a public treatment center for isolation treatment.

\section{Discussion}


The 2019-nCoV is a bete-coronavirus in the same subgenus as the Severe Acute Respiratory Syndrome (SARS), but is quite different from the Middle East Respiratory Syndrome coronavirus (MERS-CoV) ${ }^{[2]}$. Due to

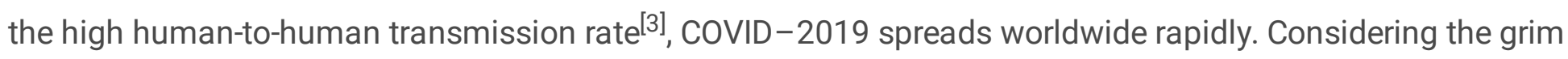
situation, the World Health Organization (WHO) declared the global outbreak of 2019-nCoV constitutes a public health emergency of international concern (PHEIC) on January 31, 2020.

Currently, the incubation period for COVID-19 pneumonia is thought to be within 14 days following exposure, with most cases at approximately 3-7 days after exposure ${ }^{[4]}$. The most important criteria for diagnoses of COVID-2019 pneumonia is positive RT-PCR results, which could be performed on the blood, body fluids, nasopharyngeal and oropharyngeal swab囚sputum『bronchoalveolar lavage and stool specimens. It has been reported that only when there are negative results of RT-PCR testing for 2019-nCoV on two specimens collected $\geq 24$ hours apart, a patient can release from quarantine $e^{[4]}$. However, the accuracy of RTPCR test of any virus would not be $100 \%$. In other words, false negative would be inevitable in some clinical cases. The accuracy is related to the severity and stage of the patients' disease. According to the current,patients with clinical manifestations and pneumonia confirmed imaging tests can be clinically diagnosed as COVID-2019 pneumonia in Hubei Province ${ }^{[5]}$. Therefore, we still highly suspect this patient has COVID-2019 pneumonia regardless of his multiple negative RT-PCR test results. When the patients are in the early or recovery stage of the disease, the detection rate of 2019-nCoV tests are also probably lower. Sometimes, laboratory errors, wrong specimen type or virus detection kit can also be the cause of false negative test results. The most preferred specimens is nasopharyngeal swab, followed by oropharyngeal swab, then sputum. Now it is also possible to improve the positive rate by collecting nasopharyngeal swab and oropharyngeal swab in the same specimen collection tube ${ }^{[6]}$.

In summary, when patients show the characteristic clinical manifestations of COVID-2019 pneumonia and chest CT typical for COVID-2019 pneumonia, and has recent history of exposure to people came back from Hubei Province, COVID-2019 should be highly suspected and appropriate treatment and isolation are still needed, even when the RT-PCR results repeatedly to be negative up to five times.

\section{Abbreviations}

RT-PCR = reverse transcriptase polymerase chain reaction

2019-nCoV = 2019 novel coronavirus

COVID-2019 = 2019 novel coronavirus disease

\section{Declarations}

Ethics approval and consent to participate

Not applicable.

Consent for publication

Page $3 / 6$ 
The patient written informed consent for their personal or clinical details along with any identifying images to be published in this study.

Availability of data and materials

All data generated or analyzed during this study are included in this published manuscript. There are no datasets related to this case report.

Competing interests

The authors have no potential conflicts of interest to disclose.

Funding

No funding received.

Authors' contributions

CLM and SY performed the majority of the sampling and testing of specimens, WYH and LY collected the case clinical data; LH drafted the manuscript, LH and TYJ participated in the design of the study; TYJ and FJT critically reviewed the manuscript. All of the authors read and approved the final manuscript.

\section{Acknowledgements}

Not applicable.

\section{References}

1. World Health Organization. Director-General's remarks at the media briefing on 2019-nCoV on 11 February 2020. https://www.who.int/dg/speeches/detail/who-director-general-s-remarks-at-the-mediabriefing-on-2019-ncov-on-11-february-2020 (Accessed on February 12, 2020).

2. Lu R,Zhao X,Li J,et al. Genomic characterization and epidemiology of 2019 novel coronavirus: implications for virus origins and receptor binding[J].Lancet 2020.

3. Li Q,Guan X,Wu P,et al.Early Transmission Dynamics in Wuhan, China, of Novel Coronavirus-Infected Pneumonia[J].N Engl J Med. 2020.

4. China National Health Commission. Diagnosis and treatment of pneumonitis caused by new coronavirus (trial version 6). Beijing: China National Health Commission, 2020.http://www.nhc.gov.cn/xcs/zhengcwj/202002/8334a8326dd94d329df351d7da8aefc2.shtml (accessed Feb 18, 2020).

5. China National Health Commission. Diagnosis and treatment of pneumonitis caused by new coronavirus (trial version 6). Beijing: China National Health Commission,2020.

http://www.nhc.gov.cn/yzygj/s7653p/202002/3b09b894ac9b4204a79db5b8912d4440.shtml(accessed Feb,5 2020). 
6. Chinese Society of Laboratory Medicine. Consensus on detection of nucleic acids in novel coronavirus pneumonia [J]. National Medical Journal of China,2020,100( 00): E003-E003.

\section{Figures}
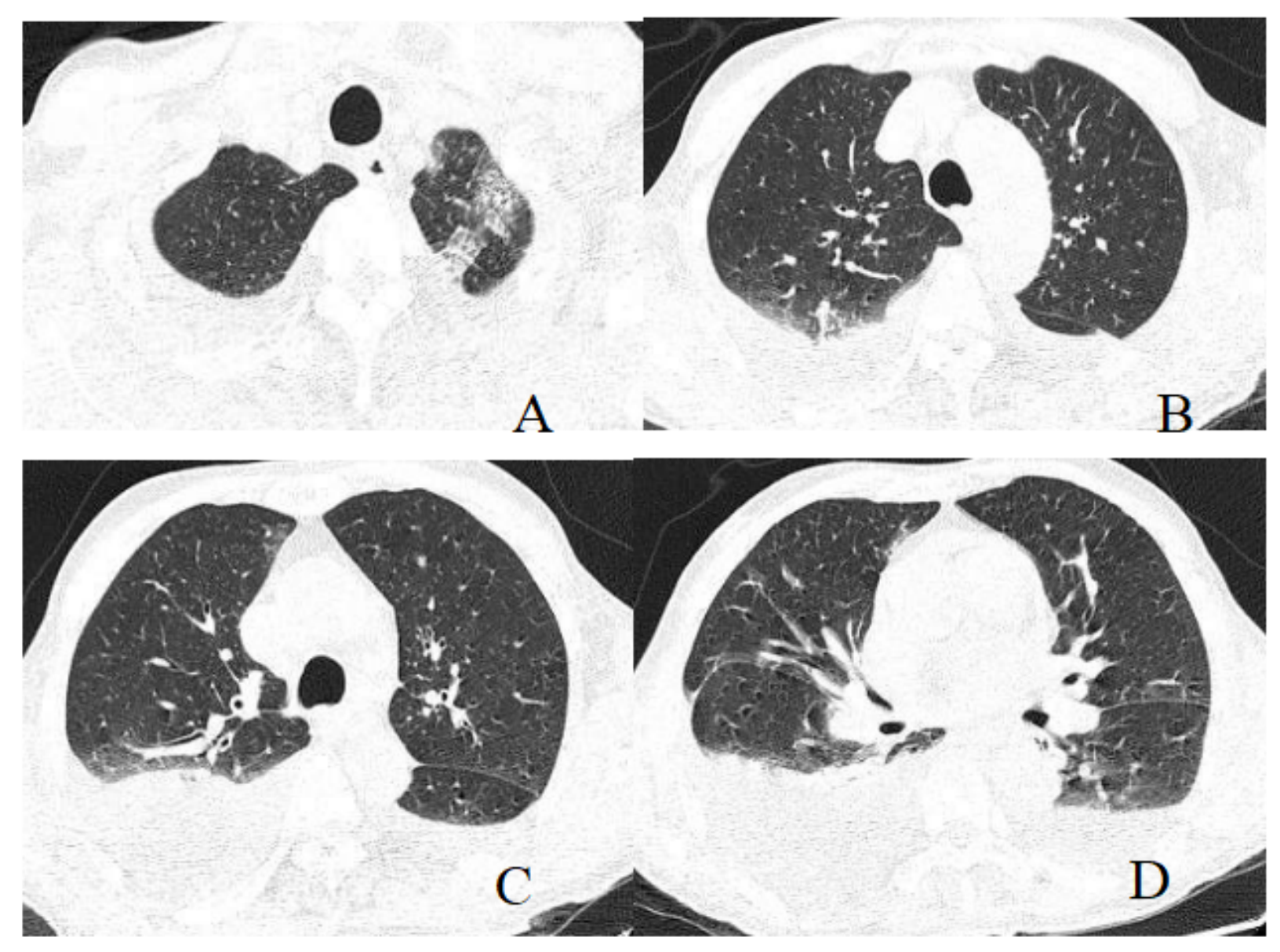

\section{Figure 1}

Chest CT scan obtained at admission. CT images show ground-glass opacities in left upper lung, bilateral pleural effusion and pericardial effusion. 


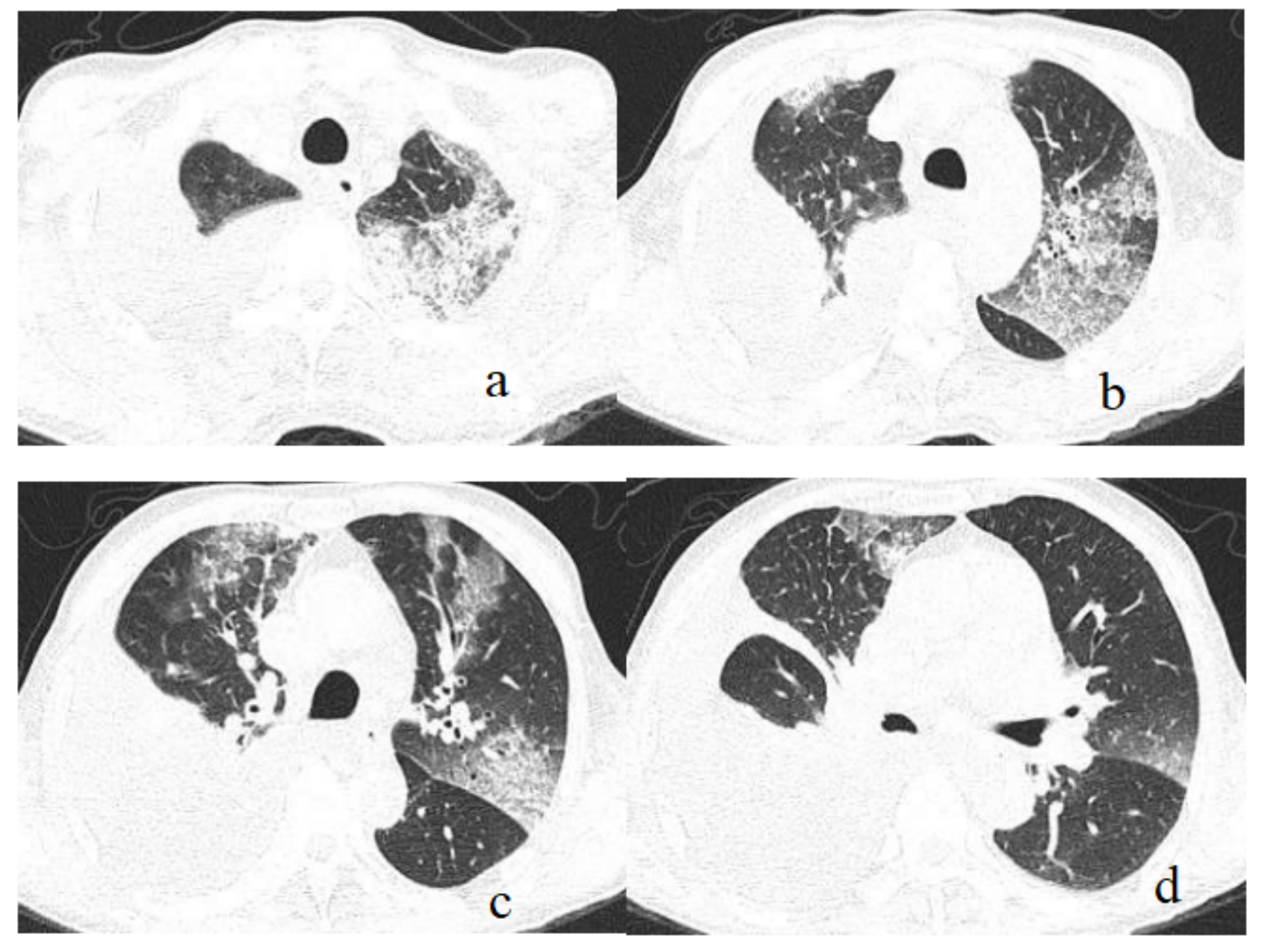

Figure 2

Chest CT scan obtained 13 days after admission. The chest CT showed multi-focal ground glass opacity, predominantly involving in left upper lung, right upper lobe and right lower lobe.

\section{Supplementary Files}

This is a list of supplementary files associated with this preprint. Click to download.

- CAREchecklistEnglish2013.docx 